\title{
Near-Field Second-Harmonic Generation Induced by Local Field Enhancement
}

\author{
A. Bouhelier, M. Beversluis, A. Hartschuh, and L. Novotny* \\ The Institute of Optics, University of Rochester, Rochester, New York 14627
}

(Received 8 March 2002; published 10 January 2003)

\begin{abstract}
The field near a sharp metal tip can be strongly enhanced if irradiated with an optical field polarized along the tip axis. We demonstrate that the enhanced field gives rise to local second-harmonic ( $\mathrm{SH}$ ) generation at the tip surface thereby creating a highly confined photon source. A theoretical model for the excitation and emission of SH radiation at the tip is developed and it is found that this source can be represented by a single on-axis oscillating dipole. The model is experimentally verified by imaging the spatial field distribution of strongly focused laser modes.
\end{abstract}

DOI: 10.1103/PhysRevLett.90.013903

PACS numbers: $42.65 . \mathrm{Ky}, 07.79 . \mathrm{Fc}, 42.50 . \mathrm{Hz}$

Field enhancement near nanoscale metal structures plays a central role for optical phenomena such as surface enhanced Raman scattering (SERS) [1], secondharmonic (SH) generation [2], and near-field microscopy [3-5]. The enhancement originates from a combination of the electrostatic lightning-rod effect, which is due to the geometric singularity of sharply pointed structures, and localized surface plasmon resonances which depend sensitively on the excitation wavelength. In most studies, the field enhancement factor is either deduced from theoretical calculations or from ensemble averaged measurements. However, recent spatially resolved SERS measurements on single molecules have shown that the field enhancement may vary by many orders of magnitude between different sites of a colloidal metal surface $[6,7]$. In order to understand which structures render particularly strong enhancements it is necessary to perform measurements on a single particle level. In this Letter, we demonstrate that local SH generation provides a direct means to determine the field enhancement near a nanoscale structure such as a sharp metal tip.

SH generation in the context of near-field optical microscopy has been discussed by various authors [8-11] but the strong dependence on local field enhancement has not been analyzed. Here, we show that the field enhancement effect localizes SH generation to the very end of a metal tip thereby creating a highly confined photon source. We experimentally demonstrate the strong light confinement and investigate the radiation properties of the SH light. We also show that the tip acts as a local probe for fields polarized along the tip axis (longitudinal fields). This unique property can be used for the mapping of field distributions in optical fields as demonstrated recently with trapped ions [12] and single molecules [13,14].

The field enhancement effect strongly depends on the polarization properties of the exciting radiation. A strong enhancement requires a polarization component along the tip axis which is not provided by a side illumination with a weakly focused Gaussian beam. However, some tightly focused higher-order laser beams give rise to strong longitudinal fields and therefore provide the neces- sary polarization conditions for strong field enhancement [11]. In Fig. 1(a) we show the calculated electric intensity distribution $\left(|\mathbf{E}(\mathbf{r}, \omega)|^{2}\right)$ near a gold tip located above a glass substrate and irradiated by an on-axis focused Hermite-Gaussian $H G_{10}$ laser mode. The calculation was based on a combination of angular spectrum and multiple multipole (MMP) method [15] and the following parameters were used: $\lambda=830 \mathrm{~nm}, \varepsilon_{\mathrm{Au}}=-26.6+$ $1.66 i, \varepsilon_{\text {glass }}=2.3$, N.A $=1.4$. The field is strongly enhanced in front of the tip because of the longitudinal polarization in the focus of the exciting laser beam. In what follows, we demonstrate that the enhanced field generates a $\mathrm{SH}$ signal which seems to emanate from an effective dipole oriented along the tip axis.

Let $\mathbf{E}(\mathbf{r}, \omega)$ denote the solution for the electric field for a given position of the tip relative to the exciting laser beam as shown in Fig. 1(a). We find that no matter what the magnitude of the enhancement factor is, the field in
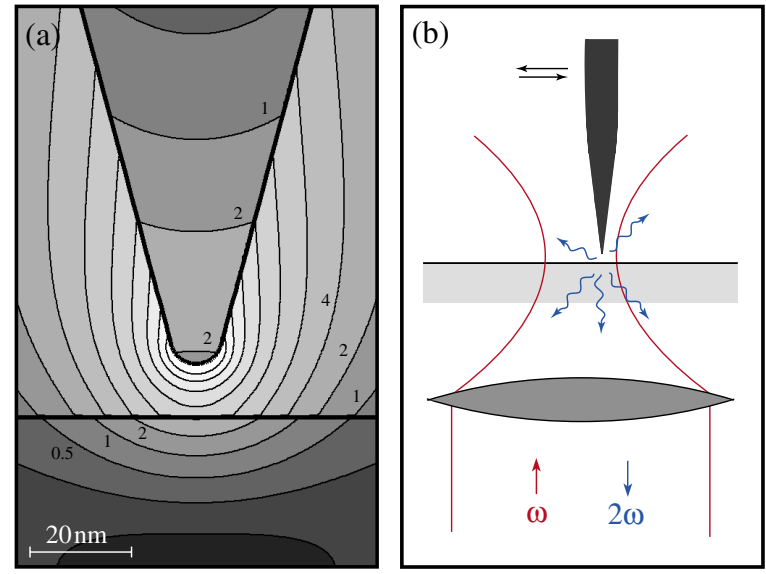

FIG. 1 (color online). (a) Calculated field distribution $\left[|E(\mathbf{r}, \omega)|^{2}\right]$ near a gold tip located above a glass substrate and irradiated by an on-axis focused $H G_{10}$ laser mode. The contour lines scale by a factor of 2. (b) Schematic of the experiment. A sharp gold tip is scanned through a tightly focused laser beam and the radiated $\mathrm{SH}$ signal is detected for each position of the tip. 
the vicinity of the tip can be quite accurately described by the fields of an effective dipole $\mathbf{p}(\omega)$ located at the center of the tip apex and with the magnitude

$$
\mathbf{p}(\omega)=\left[\begin{array}{ccc}
\alpha_{\perp} & 0 & 0 \\
0 & \alpha_{\perp} & 0 \\
0 & 0 & \alpha_{\|}
\end{array}\right] \mathbf{E}_{o}(\omega),
$$

where we chose the $z$ axis to coincide with the tip axis. $\mathbf{E}_{o}$ is the exciting electric field in the absence of the tip. The transverse polarizability $\alpha_{\perp}$ is identical to the quasistatic polarizability of a small sphere whereas the longitudinal polarizability $\alpha_{\|}$is given by

$$
\alpha_{\|}(\omega)=8 \pi \varepsilon_{o} r_{o}^{3} f_{e}(\omega) .
$$

Here $r_{o}$ is the tip radius and $f_{e}$ the complex field enhancement factor. For a wavelength of $\lambda=830 \mathrm{~nm}$, a gold tip with $\varepsilon=-26.6+1.66 i$ and a tip radius of $r_{o}=10 \mathrm{~nm}$, our numerical calculations based on the multiple multipole method [15] lead to $f_{e}=-2.9+11.8 i$. The expression for $\alpha_{\|}$originates from the requirement that the magnitude of the field produced by $\mathbf{p}(\omega)$ at the surface of the tip is equal to the computationally determined field which we set equal to $f_{e} \mathbf{E}_{o}$. The electric field $\mathbf{E}$ in the vicinity of the tip is now approximated as

$$
\mathbf{E}(\mathbf{r}, \omega)=\mathbf{E}_{o}(\mathbf{r}, \omega)+\frac{1}{\varepsilon_{o}} \frac{\omega^{2}}{c^{2}} \mathcal{G}\left(\mathbf{r}, \mathbf{r}_{o}, \omega\right) \mathbf{p}(\omega),
$$

where $\mathbf{r}_{o}$ specifies the origin of $\mathbf{p}$ and $G$ is the free space dyadic Green's function. SH generation at the tip originates mainly from a surface nonlinear polarization $\mathbf{P}^{s}$ $[2,16]$. This polarization is caused by the electric field component along the surface normal which varies rapidly across the interface layer. Despite its nonlocal origin, the interface nonlinearity can be represented by a local surface nonlinear susceptibility $\chi^{s}$ [17]. The strongest contribution to $\mathbf{P}^{s}$ is due to the electric field components normal to the tip surface

$$
P_{n}^{s}\left(\mathbf{r}^{\prime}, 2 \omega\right)=\chi_{n n n}^{s}(-2 \omega ; \omega, \omega) E_{n}^{(\mathrm{vac})}\left(\mathbf{r}^{\prime}, \omega\right) E_{n}^{(\mathrm{vac})}\left(\mathbf{r}^{\prime}, \omega\right)
$$

where the index $n$ denotes the surface normal, $\mathbf{r}^{\prime}$ a point on the surface of the tip, and the superscript "vac" indicates that the fields are evaluated on the vacuum side of the surface. The field at the second-harmonic frequency is calculated as

$\mathbf{E}(\mathbf{r}, 2 \omega)=\frac{1}{\varepsilon_{o}} \frac{(2 \omega)^{2}}{c^{2}} \int_{\text {surface }} G\left(\mathbf{r}, \mathbf{r}^{\prime}, 2 \omega\right) \mathbf{P}_{\mathrm{eff}}^{s}\left(\mathbf{r}^{\prime}, 2 \omega\right) d^{2} \mathbf{r}^{\prime}$,

where the effective surface polarizability is defined as

$$
\mathbf{P}_{\text {eff }}^{s}=G^{-1} G_{\text {tot }} \mathbf{P}^{s} \text {. }
$$

$G_{\text {tot }}$ is the total Green's function of the system "free-space + tip." It accounts for the interaction of the surface polarization currents with the metal tip and it contains all electronic resonances at $2 \omega$. Although the tip is infinite in size the field enhancement effect localizes $\mathrm{SH}$ generation to the very end of the tip. Therefore, we expand Eq. (5) into a multipole series with respect to the center of the tip apex $\mathbf{r}_{o}$. Furthermore, because of the small tip size we retain only the lowest dipole order and obtain

$$
\mathbf{E}(\mathbf{r}, 2 \omega)=\frac{1}{\varepsilon_{o}} \frac{(2 \omega)^{2}}{c^{2}} \mathcal{G}\left(\mathbf{r}, \mathbf{r}_{o}, 2 \omega\right) \mathbf{p}_{\mathrm{eff}}(2 \omega),
$$

where the effective dipole moment $\mathbf{p}_{\text {eff }}$ expressed in terms of $\mathbf{r}^{\prime \prime}=\mathbf{r}^{\prime}-\mathbf{r}_{o}$ is

$$
\mathbf{p}_{\text {eff }}(2 \omega)=\int_{\text {surface }} \mathbf{P}_{e f f}^{s}\left(\mathbf{r}_{o}+\mathbf{r}^{\prime \prime}, 2 \omega\right) d^{2} \mathbf{r}^{\prime \prime} .
$$

Notice that $\mathbf{p}_{\text {eff }}$ depends on the relative position of tip and excitation field. Furthermore, through $G_{\text {tot }}$ the effective dipole becomes frequency dependent in a nontrivial way [18].

Here, we experimentally demonstrate that $\mathrm{SH}$ generation at an infinite sharp tip can indeed be represented by a dipole oscillating at the second-harmonic frequency. Because of the field enhancement effect, SH generation is dominated by excitation fields polarized along the tip axis. Therefore, the tip acts as a probe for longitudinal fields. This is different for a small spherical particle which is equally sensitive to all fields.

The experimental arrangement is shown schematically in Fig. 1(b). The excitation field is provided by a modelocked Ti:sapphire laser rendering $140 \mathrm{fs}$ light pulses at a wavelength of $830 \mathrm{~nm}$. The beam is spatially filtered in order to produce a well-defined Gaussian beam $\left(H G_{00}\right)$. The beam is then reflected by a dichroic beam splitter and is focused onto the surface of a glass cover slip by an oil immersion microscope objective (NA:1.4). Alternatively, the edge of a $180^{\circ}$ phase plate can be placed in the beam path to produce a Hermite-Gaussian mode $\left(H G_{10}\right)$ [11]. The radius of our chemically etched gold tip was verified by scanning electron microscopy and was approximatively $10 \mathrm{~nm}$ with a cone angle of $30^{\circ}$. The tip was positioned above the glass surface and held at close proximity by means of shear-force regulation [19]. The tip is raster scanned through the focal spot using piezoelectric actuators. The SH signal emitted by the tip is collected by the same objective, passed through the dichroic and a notch filter, and directed on an avalanche photodiode. Correlation of the tip position with the detected SH signal allows us to reconstruct a two-dimensional image of the field distribution. Typical average excitation powers are on the order of $500 \mu \mathrm{W}$ which corresponds to an average intensity of roughly $500 \mathrm{~kW} / \mathrm{cm}^{2}$ and a peak intensity of $2.5 \mathrm{GW} / \mathrm{cm}^{2}$ in the laser focus. Based on the radiated SH power and the calculated enhancement factor we estimate the surface susceptibility to be on the order of $\chi^{s} \approx$ $10^{-31} \mathrm{Cb} \mathrm{m} / \mathrm{V}^{2}$. 

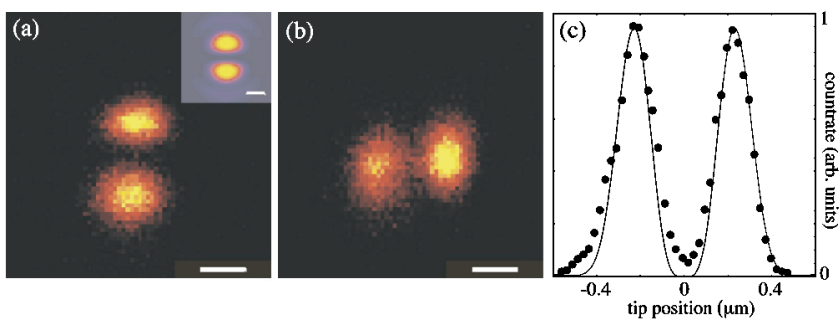

FIG. 2 (color). Tip-induced SH image of the focal fields of a strongly converging Gaussian $\left(H G_{00}\right)$ beam. The two lobes are oriented in the direction of the incident polarization [rotated by $90^{\circ}$ between (a) and (b)]. The inset shows the calculated longitudinal field distribution $\left(E_{o, z}^{2}\right)$ of a focused $H G_{00}$ beam just above the glass surface. (c) Comparison of $E_{o, z}^{4}$ (solid line) with the experimentally detected $\mathrm{SH}$ signal (dots). Scale bars: $250 \mathrm{~nm}$.

Figures 2(a) and 2(b) show SH scanned images of the focal spot of a Gaussian $H G_{00}$ beam for two perpendicular polarizations. Two intense lobes oriented along the polarization direction can be recognized. The inset in Fig. 2(a) shows the calculated image of the longitudinal field $\left(E_{o, z}^{2}\right)$ in the focus of a Gaussian beam for one of the two polarizations. The calculation of the focal fields is based on an angular spectrum representation using the Fresnel reflection and transmission coefficients at the interface $[14,20]$. The experimental image agrees very well with the calculated field distribution, indicating that the longitudinal field is indeed the main excitation source for $\mathrm{SH}$ generation at the gold tip. Figure 2(c) compares the $\mathrm{SH}$ excitation field $\left(E_{o, z}^{4}\right)$ with the experimentally detected SH signal for a single line scan through the laser focus along the direction of the two longitudinal field lobes. No fitting was performed besides scaling the experimental and theoretical curves to the same maximum value. The good agreement indicates that $\mathrm{SH}$ generation is almost exclusively induced by the enhanced longitudinal field at the tip's surface.

The experiment was repeated by focusing a HermiteGaussian $H G_{10}$ beam. Figure 3(a) shows the resulting $\mathrm{SH}$ image. The detected pattern is now characterized by a three-lobe pattern. Most of the energy is found on the center lobe which is bordered by two weaker side lobes aligned in the direction of the incident polarization. The inset shows the calculated longitudinal field distribution $\left(E_{o, z}^{2}\right)$. The slight rotation of the pattern originates from the initial mode conversion. Figure 3(b) compares the experimental $\mathrm{SH}$ pattern with the calculated $\mathrm{SH}$ excitation field $\left(E_{o, z}^{4}\right)$ along a line through the center of the beam. Again, no fitting was performed besides scaling the experimental and theoretical curves to the same maximum value.

The major deviation between the recorded SH signal and the calculated value of $E_{o, z}^{4}$ is observed near the minima of the curves in Figs. 2(c) and 3(b). The origin of this deviation can be attributed mainly to $\mathrm{SH}$

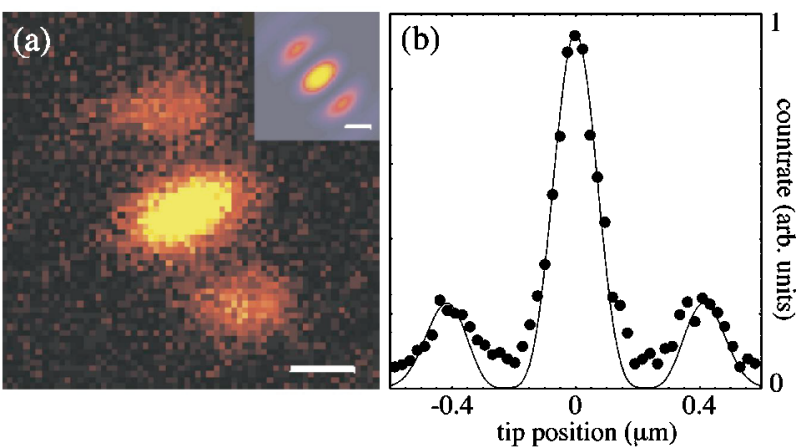

FIG. 3 (color). (a) Tip-induced SH image of the focal fields of a strongly converging Hermite-Gaussian $\left(H G_{10}\right)$ beam. The inset shows the calculated longitudinal field distribution $\left(E_{o, z}^{2}\right)$ of a focused $H G_{10}$ beam. (b) Comparison of $E_{o, z}^{4}$ (solid line) with the experimentally detected $\mathrm{SH}$ signal (dots). Scale bars: $250 \mathrm{~nm}$.

generation by transverse excitation fields. A detailed calculation using the above theory shows that the inclusion of the transverse fields lifts the minima in the theoretical curves and therefore provides a better fit to the experimental data. However, the stronger the field enhancement factor is the weaker the contribution of the transverse fields will be. Therefore, the ratio between maxima and minima in the experimental data is a direct measure for the field enhancement factor. For sufficiently strong enhancement, SH generation originates mainly from the enhanced electric field component along the tip axis as shown by our data. Thus, the tip acts as a sensor for longitudinal field components.

To demonstrate that the sensitivity to longitudinal fields is unique to the elongated geometry of the tip we dispersed a colloidal solution of $30 \mathrm{~nm}$ gold spheres on a clean glass cover slip. As with the previous set of experiments, spatially isolated particles were scanned through the laser foci of the $H G_{00}$ and $H G_{10}$ beams and the generated SH signal was detected to create an image. The resulting scan images are shown in Fig. 4. For the case of a Gaussian $H G_{00}$ excitation beam the pattern now consists of a single lobe elongated in direction of the incident polarization. The elongated pattern originates from the particle's sensitivity to the total field strength $\left(E_{o}^{2}\right)$ as opposed to the longitudinal field strength $\left(E_{o, z}^{2}\right)$. The transverse contribution $\left(E_{o, x}^{2}+E_{o, y}^{2}\right)$ is a circular spot with a Gaussian intensity profile, and the longitudinal contribution is composed of two lobes oriented along the incident polarization direction as demonstrated previously. The inset in Fig. 4(a) shows the calculated intensity pattern taking into account the effect of all the components of the electromagnetic field. The $\mathrm{SH}$ image depicted in Fig. 4(b) originates from scanning the same gold particle through the focus of the $H G_{10}$ beam. The pattern is similar to the pattern shown in Fig. 3(a) suggesting that the focal field distribution of a 

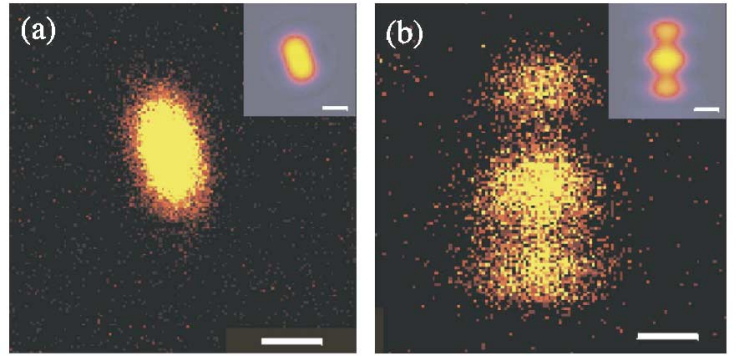

FIG. 4 (color). SH image of the focal fields of a strongly converging Gaussian $H G_{00}$ beam (a) and a Hermite-Gaussian $H G_{10}$ beam (b) obtained by replacing the tip with a spherical gold particle. The insets show the calculated total field distributions $\left(E_{o}^{2}=E_{o, x}^{2}+E_{o, y}^{2}+E_{o, z}^{2}\right)$ just above the glass surface. Scale bars: $250 \mathrm{~nm}$.

Hermite-Gaussian beam is dominated by the longitudinal field.

Finally, we demonstrate that the radiation pattern of the emitted SH light is identical to the radiation pattern of a dipole oriented along the tip axis. A dipole oriented vertically on a dielectric substrate generates a rotationally symmetric radiation pattern which, in the dielectric halfspace, is strongly peaked at the critical angle of total internal reflection $\theta_{c}$ [21,22]. As shown in Fig. 5, we obtain a similar radiation pattern for the $\mathrm{SH}$ light generated by the tip. The tip has been positioned into a longitudinal field lobe of the illuminating Gaussian beam and the emission pattern of the generated SH light has been directed on a CCD camera. The recorded radiation pattern shown in Fig. 5 consists of a bright ring emitted near the critical angle $\left(\sim 41^{\circ}\right)$ as expected from the emission symmetry of a vertically aligned dipole. The slight intensity variations originate from deviations of the tips rotational symmetry.

In conclusion, longitudinal fields created by strongly focused beams are responsible for the field enhancement at the end of a sharp metallic tip. The enhancement generates locally a SH source which can be accurately

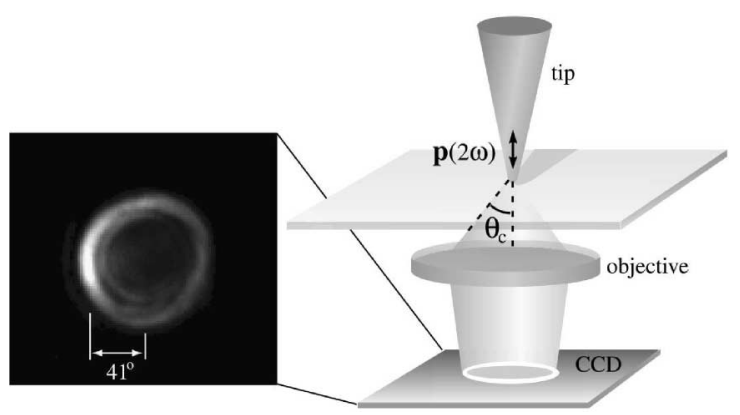

FIG. 5. SH emission pattern from a gold tip. The tip produces a nearly conical radiation pattern peaked at the critical angle of total internal reflection $\theta_{c}$. The pattern is identical to the emission pattern of a dipole oriented vertically on a dielectric substrate. modeled by a single on-axis oscillating dipole. The dipole strength is a direct measure for the field enhancement factor. The SH source could further be explored for more dedicated experiments such as absorption spectroscopy of nanostructures. Because of the strong optical nonlinearities of noble metals it is reasonable to assume that second-harmonic generation will be dominated by the tip and not by the sample as long as nonmetallic structures are investigated.

The authors wish to acknowledge stimulating discussions with J-J. Greffet and J. Zurita-Sanchez. This work was funded by the U.S. Department of Energy through Grant No. DE-FG02-01ER15204 and partially supported by the Swiss National Foundation (A. B.).

*Electronic address: novotny@optics.rochester.edu

[1] For a review, see A. Otto, I. Mrozek, H. Grabhorn, and W. Akemann, J. Phys. Condens. Matter 4, 1143 (1992).

[2] C. K. Chen, T. F. Heinz, D. Ricard, and Y. R. Shen, Phys. Rev. B 27, 1965 (1983).

[3] J. Wessel, J. Opt. Soc. Am. B 2, 1538 (1985).

[4] E. J. Sànchez, L. Novotny, and X. Xie, Phys. Rev. Lett. 82, 4014 (1999).

[5] J. L. Bohn, D. J. Nesbitt, and A. Gallagher, J. Opt. Soc. Am. A 18, 2998 (2001).

[6] K. Kneipp, Y. Wang, H. Kneipp, I. Itzkan, R. R. Dasary, and M. S. Feld, Phys. Rev. Lett. 78, 1667 (1997).

[7] S. Nie and S. R. Emory, Science 275, 1102 (1997).

[8] A.V. Zayats et al., Phys. Rev. B 61, 4545 (2000).

[9] S. I. Bozhevolnyi and V. Z. Lozovski, Phys. Rev. B 65, 235420 (2002).

[10] I. I. Smolyaninov, H. Y. Liang, C. H. Lee, and C. C. Davis, J. Appl. Phys. 89, 206 (2001).

[11] L. Novotny, E. J. Sànchez, and X. Xie, Ultramicroscopy 71, 21 (1998).

[12] G. R. Guthöhrlein, M. Keller, K. Hayasaka, W. Lange, and H. Walther, Nature (London) 414, 49 (2001).

[13] B. Hecht, B. Sick, and L. Novotny, Phys. Rev. Lett. 85, 4482 (2000).

[14] L. Novotny, M. R. Beversluis, K. S. Youngworth, and T. G. Brown, Phys. Rev. Lett. 86, 5251 (2001).

[15] Ch. Hafner, The Generalized Multiple Multipole Technique for Computational Electromagnetics (Artech, Boston, 1990).

[16] P. Guyot-Sionnest, W. Chen, and Y. R. Shen, Phys. Rev. B 33, 8254 (1986).

[17] B. S. Mendoza and W. Mochàn, Phys. Rev. B 53, 4999 (1996), and references therein.

[18] J. A. Porto, R. Carminati, and J.-J. Greffet, J. Appl. Phys. 88, 4845 (2000).

[19] K. Karrai and D. R. Grober, Appl. Phys. Lett. 66, 1842 (1995).

[20] D. P. Biss, and Th. G. Brown, Opt. Express 9, 490 (2001).

[21] W. Lukosz and R. E. Kunz, J. Opt. Soc. Am. 67, 1615 (1977).

[22] A. P. Bartko and R. M. Dickson, J. Phys. Chem. B 103, 11237 (1999). 